Research Paper:

\title{
Anticonvulsive and Antioxidant Effects of Pioglitazone on Pentylenetetra- zole-induced Seizures in Rats
}

\author{
Yasaman Ghiasi ${ }^{1}$ (1), Saba Rostamian ${ }^{1}$, Ehsan Aali ${ }^{2},{ }^{*}$ Yazdan Naderi ${ }^{2}$ (1)
}

1. Student Research Committee, Qazvin University of Medical Sciences, Qazvin, Iran.

2. Department of Pharmacology, School of Medicine, Qazvin University of Medical Sciences, Qazvin, Iran

\begin{tabular}{|l|l|l}
\hline $\begin{array}{c}\text { Use eour device toscan } \\
\text { and read the artice online }\end{array}$ & $\begin{array}{l}\text { Citation Ghiasi Y, Rostamian S, Aali E, Naderi Y. Anticonvulsive and Antioxidant Effects of Pioglitazone on Pentylenetetrazole-Induced } \\
\text { Seizures in Rats. Journal of Inflammatory Diseases. 2020; 24(4):320-331. https://doi.org/10.32598/JQUMS.24.4.6 }\end{array}$ \\
dol'https://doi.org/10.32598/JQUMS.24.4.6
\end{tabular}

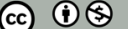

Received: 10 Aug 2020

Accepted: 30 Sep 2020

Available Online: 01 Oct 2020

Keywords:

Seizure, Pioglitazone,

Pentylenetetrazole,

Antioxidant

Anticonvulsant

\section{ABSTRACT}

Background Epilepsy is a neurologic dysfunction caused by abnormal electrical activity in the brain. Oxidative stress is involved in the seizure-induced brain damage.

Objective This study aimed to evaluate the anticonvulsant and antioxidant effects of pioglitazone (a peroxisome proliferator-activated receptor gamma agonist used for treatment of type 2 diabetes) on Pentylenetetrazole (PTZ)-induced seizure in rats.

Methods In this experimental study, 28 rats weighing 20-30 g were divided into four groups of control, pioglitazone, PTZ, and treatment. For treatment, PTZ $(85 \mathrm{mg} / \mathrm{kg})$ or normal saline was injected intraperitoneally and 4 hours later, pioglitazone $(80 \mathrm{mg} / \mathrm{kg})$ was administrated orally. Carboxymethylcellulose was administered orally in the control and PTZ groups, instead of pioglitazone. One hour after PTZ injection, seizure severity was assessed using Racine scale. Then, the rats were decapitated and the Malondialdehyde (MDA) level and the activity of Catalase (CAT) and Superoxide Dismutase (SOD) in their hippocampus samples were measured by standard methods.

Findings Pioglitazone administration significantly increased the latency to the onset of seizure stages 1-4 and prevented the stage 5 . It significantly reduced the lipid peroxidation caused by PTZ-induced seizure and increased the activity of CAT and SOD enzymes in the hippocampus of rats.

Conclusion Antioxidant effects of pioglitazone may play a role in preventing stable PTZ-induced seizures and protecting neurons from seizure-caused damage.

\section{Extended Abstract}

\section{Introduction}

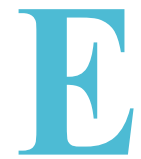

pilepsy is a chronic neurological disorder in the brain characterized by recurrent seizures [1]. The severity of these attacks varies from mild episodes involving involuntary movements of a part of the body, to veloped countries, the prevalence of the disease is $40-70$ per 100,000 people, while in developing countries the rate is $100-190$ per 100,000 people [3]. More than $60 \%$ of people with epilepsy need treatment with anticonvulsant drugs [4]. Various studies have shown that seizures cause neuronal damage and complications through the production of free radicals and oxidative stress $[10,11]$. Therefore, treatment with antioxidant drugs may reduce seizure damage in the brain [12]. One of the models of seizures in rodents is the general seizures involving all parts of the body $[1,2]$. In de-

\section{* Corresponding Author}

Yazdan Naderi

Address: Department of Pharmacology, School of Medicine, Qazvin University of Medical Sciences, Qazvin, Iran.

Tel: +98 (28) 33336001

E-mail: y.naderi@qums.ac.ir 
administration of high dose of Pentylenetetrazole (PTZ) [13]. PTZ damages GABAergic interneurons in the hippocampus by causing oxidative stress, resulting in the induction of stable seizures [14]. Pioglitazone is a PPAR- $\gamma$ nuclear receptor agonist used for treatment of type 2 diabetes [15]. Various studies have shown that pioglitazone has antioxidant effects [16]. Due to the role of oxidative stress in neuronal damage caused by seizures, in this study we aims to investigate the antioxidant and anticonvulsant effects of pioglitazone in a rat model of seizure induced by PTZ.

\section{Materials and Methods}

In this study, 28 adult male rats were divided into 4 groups of 7; control, pioglitazone, PTZ, and treatment. For treatment, pioglitazone $(80 \mathrm{mg} / \mathrm{kg}$ ) was administered orally and, 4 hours later, normal saline or PTZ $(85 \mathrm{mg} / \mathrm{kg}$ ) was injected intraperitoneally. Carboxymethylcellulose $(0.5 \%)$ was used instead of pioglitazone in control and PTZ groups. Within one hour after PTZ injection, the latency time to the onset of different stages of seizures (according to Racine's criteria) was measured. Racine stages include: Stage $0=$ no seizures, stage $1=$ mouth and facial movement, stage $2=$ rhythmic head nodding, stage $3=$ forelimb clonus, stage 4=rearing with forelimb clonus, and stage $5=$ rearing and falling with forelimb clonus. Rats were then anesthetized and their brains were extracted. Next, the Malondialdehyde (MDA) level and the activity of catalase and superoxide dismutase enzymes in hippocampal samples were measured. Results were expressed based on Mean \pm SEM. Student t-test was used to compare the latency time to the onset of different stages of seizures. In order to investigate the difference between the mean MDA and the activity of antioxidant enzymes in the study groups, Tukeys' post hoc test and one-way ANOVA were used, considering $\mathrm{P}<0.05$ as the significance level.

\section{Results}

The results showed that oral administration of $80 \mathrm{mg} / \mathrm{kg}$ pioglitazone or its vehicle (control group) did not induce seizures in rats; however, oral administration of $80 \mathrm{mg} / \mathrm{kg}$ pioglitazone 4 hours before intraperitoneal administration of 85 $\mathrm{mg} / \mathrm{kg}$ PTZ increased the latency time to the onset of seizures stages 1 to 4 of and prevented the stage 5 (Table 1). Measurement of MDA level in hippocampal samples showed that PTZ-induced seizures significantly increased lipid peroxidation in the hippocampus of rats $(\mathrm{P}<0.001)$. Moreover, oral administration of $80 \mathrm{mg} / \mathrm{kg}$ pioglitazone 4 hours before $85 \mathrm{mg} / \mathrm{kg}$ PTZ administration significantly reduced MDA in hippocampus samples $(\mathrm{P}<0.05)$. PTZ-induced seizures significantly reduced the activity of catalase and superoxide dismutase in the hippocampus samples compared to the control group $(\mathrm{P}<0.001)$. However, oral administration of pioglitazone 4 hours before intraperitoneal administration of PTZ significantly increased the activity of superoxide dismutase and catalase enzymes $(\mathrm{P}<0.05)$.

\section{Conclusion}

Pioglitazone administration increases the PTZ-induced seizure threshold and reduces the PTZ-induced oxidative stress in the hippocampus of rats. Therefore, pioglitazone may have antioxidant effects against PTZ-induced seizures.

\section{Ethical Considerations}

\section{Compliance with ethical guidelines}

This study was approved by the Research Ethics Committee of Qazvin University of Medical Sciences (Code: IR.QUMS.REC.1398.004).

Table 1. Effect of pioglitazone on the latency time (seconds) to the onset of different stages of PTZ-induced seizures

\begin{tabular}{cccccc}
\hline & \multicolumn{5}{c}{ Mean \pm SEM } \\
\cline { 2 - 5 } & Stage 1 & Stage 2 & Stage 3 & Stage 4 & Stage 5 \\
\hline Control & - & - & - & - & - \\
Pioglitazone $(80 \mathrm{mg} / \mathrm{kg})$ & - & - & - & - & $216.4 \pm 6.234$ \\
PTZ $(85 \mathrm{mg} / \mathrm{kg})$ & $58.44 \pm 7$ & $90.4 \pm 2.69$ & $104.82 \pm 5$ & $203.7 \pm 6.23$ & - \\
Treatment & & & & & \\
\hline
\end{tabular}

${ }^{\text {\#PTZ }}(85 \mathrm{mg} / \mathrm{kg})+$ Pioglitazone $(80 \mathrm{mg} / \mathrm{kg}) ;{ }^{*} \mathrm{P}<0.05 ; * * \mathrm{P}<0.01 ; * * * \mathrm{P}<0.001$ compared to PTZ group. 
(Previous Title: The Journal of Qazvin University of Medical Sciences)

Funding

This research received financial support from the Student Research Committee of Qazvin University of Medical Sciences.

Authors' contributions

All authors equally contributed to preparing this article.

Conflicts of interest

The authors declared no conflict of interest. 


\title{
اثرات ضدتشنج و آنتىاكسيدان بيوكَليتازون در برابر تشنج ناشى از ينتيلن تترازول در موش سورى
}

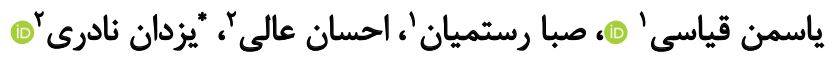 \\ 1. اكميته تحقيقات دانشجويى دانشكاه علوميزشكى قزوين، قزوين، ايران.

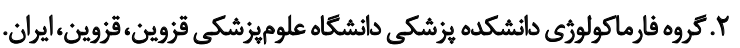

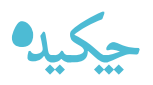

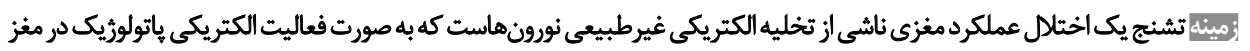

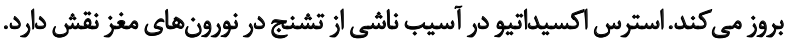

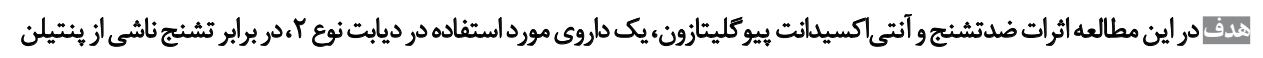

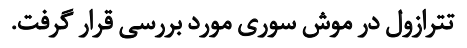

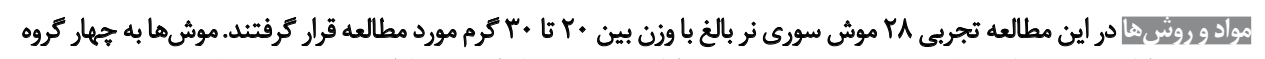

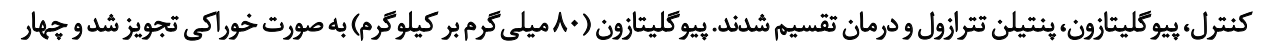

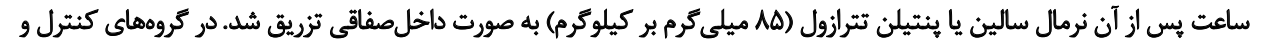

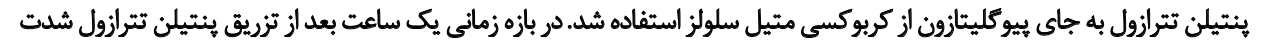

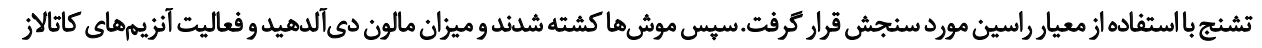

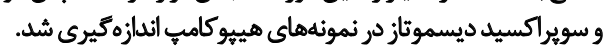

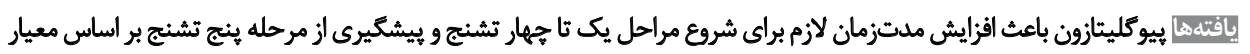

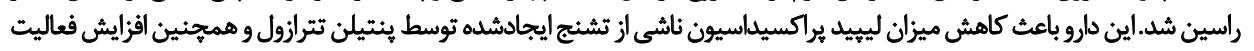

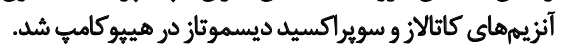

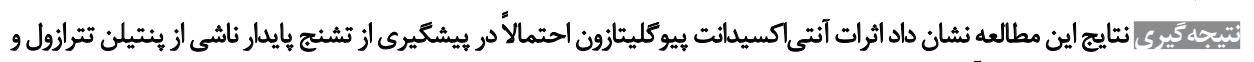

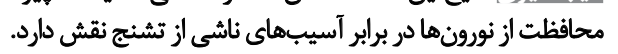

تاريخ دريافت: • م مرداد

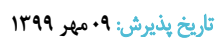

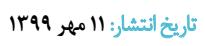

: Lotglouls

تشنج، بيوكليتازون،

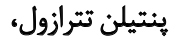
آنتيى اكسيدائت تئت

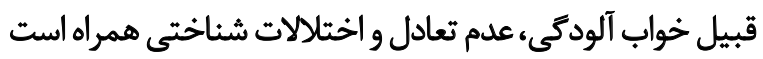

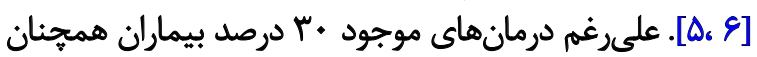

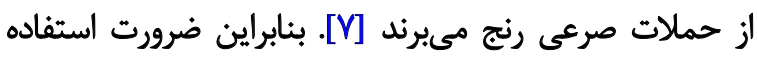

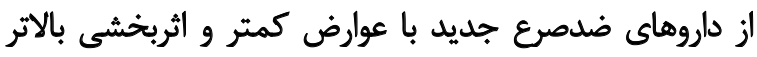

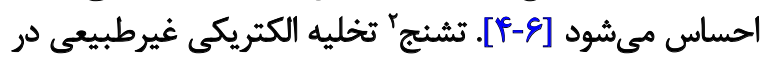

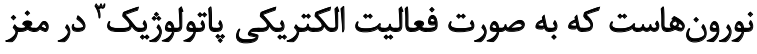

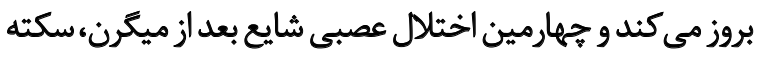

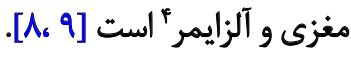

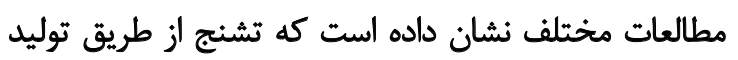

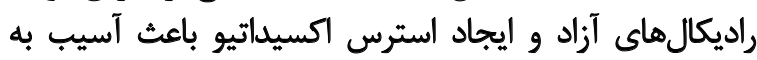

2. Seizure

3. Pathologic

4. Alzheimer

\section{드.}

dale صعُ يك اختلال عصبى مزمن در مغز است كه به صورت

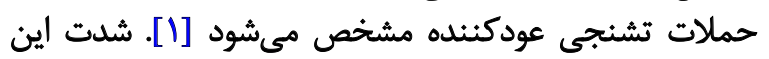

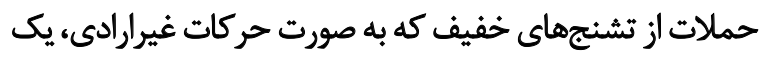

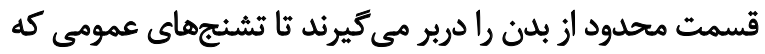

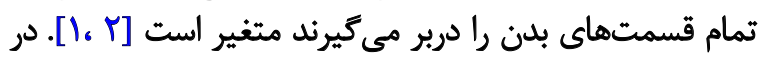

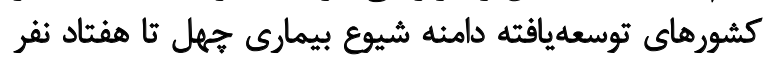

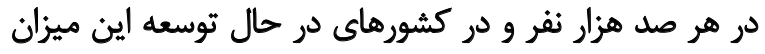

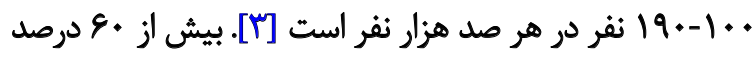

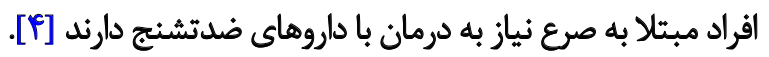

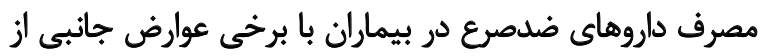

1. Epilepsy

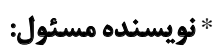

يزوان نادري

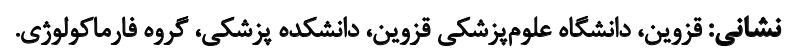
تلفن: 1 (T) راياثامه: y.naderi@qumsa.ac.ir 
تترازول: 10 • درصد CMC به صورت خوراكى به موشها دادي داده

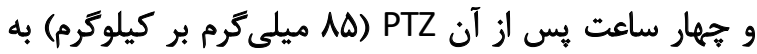

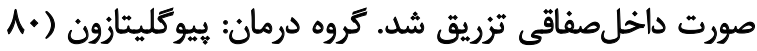

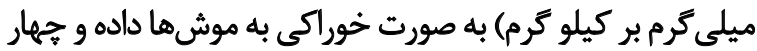

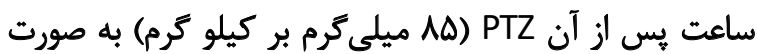
داخل صفاقى تزريق شد.

در بازه زمانى يك ساعت بعد از تزريق PTZ شدت تشنج با

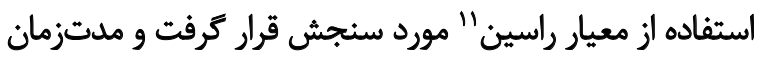

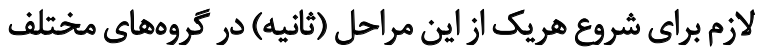

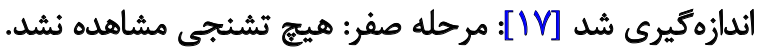

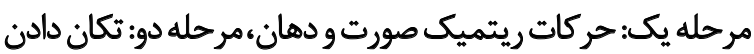

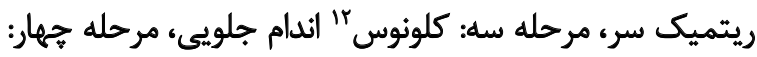

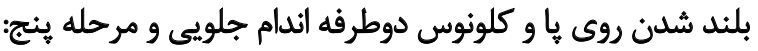

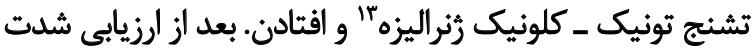

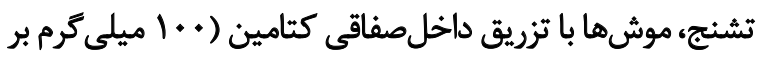

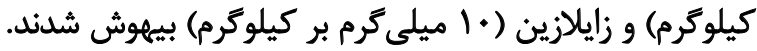

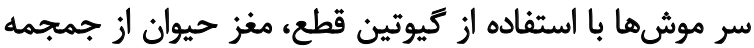

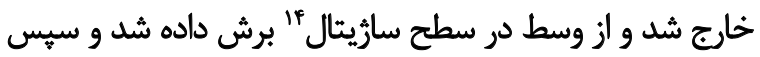

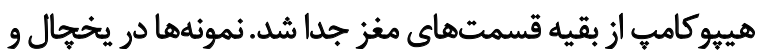

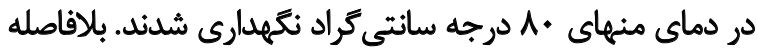

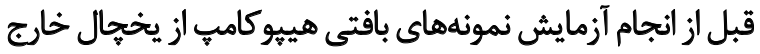

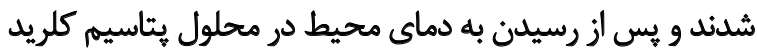

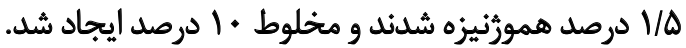

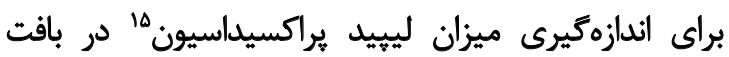

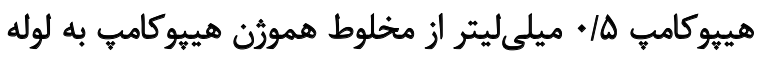

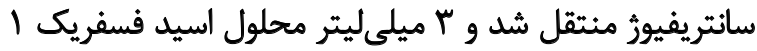

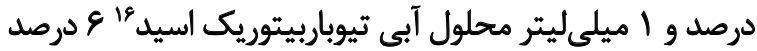

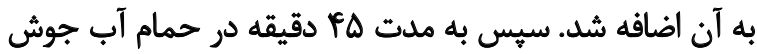

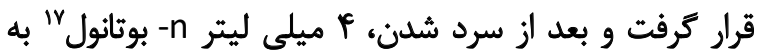

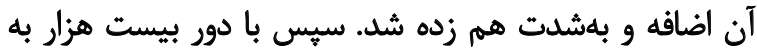

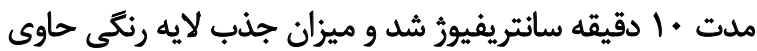

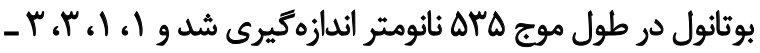

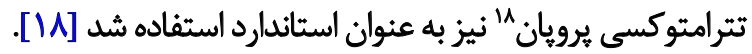

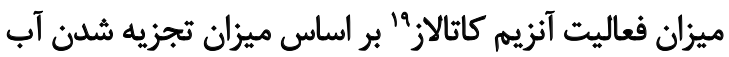

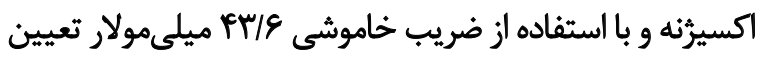
شد. بافر استخراج، حاوي بافر فسفات بافر سالين •ه ميلى خولار،

\section{Racine classification}

12. Clonus

13. Generalized tonic clonic seizure

14. Sagittal surface

15. Lipid peroxidation

16. Thiobarbituric acid

17. N-butanol

18. 1,1,3,3-tetramethoxypropane

19. Catalase
نورونها و عوارض ناشى از تشنج مي إشود [11 ، +1]. بنابراين

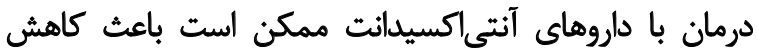

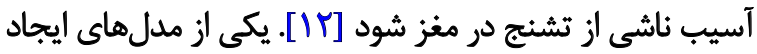

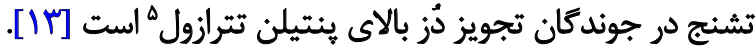

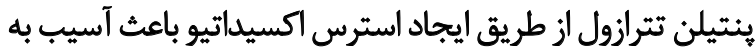

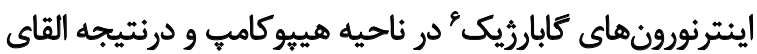

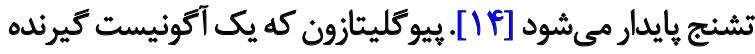

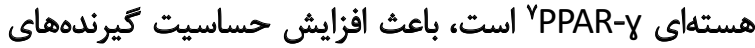

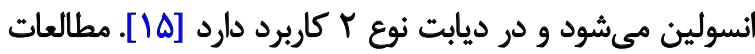

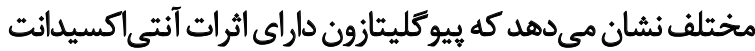

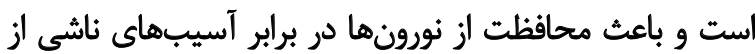

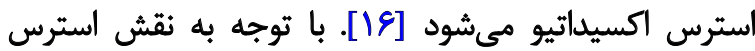

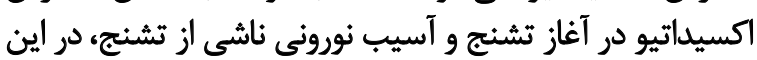

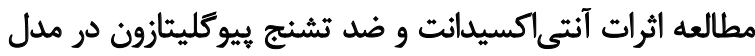
تشنج ايجادشده توسط ينتيلن تثرازول در موش هاتى سورى مورد

$$
\text { بررسى قرار كرفت. }
$$

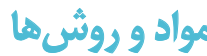

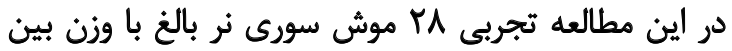

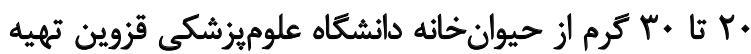

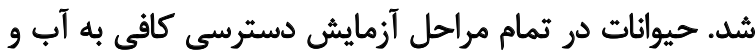

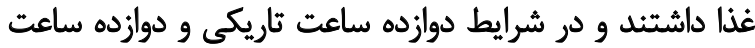

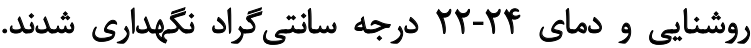

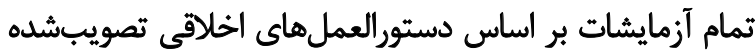

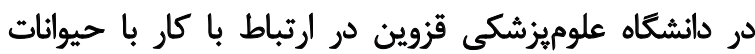

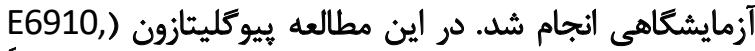

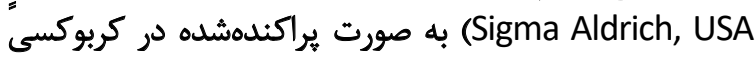

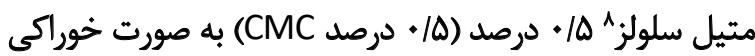

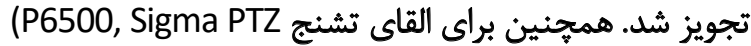
Aldrich, USA)

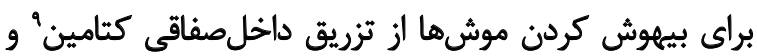

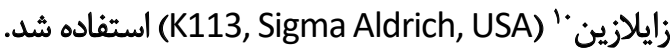

موشهاى سورى مورد مطالعه به جهار گروه تقسيم شدون

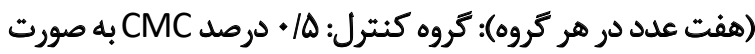

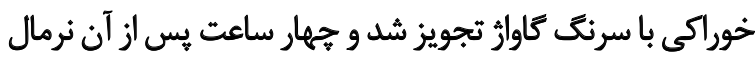

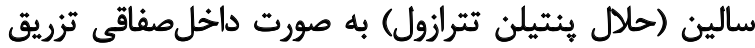

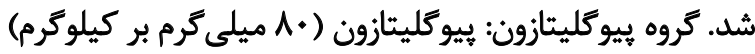

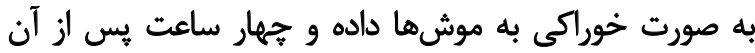
نرمال سالين به صورت داخلصفاقي تزوريق شد. كروه ينتيلن

5. Pentylenetetrazol (PTZ)
6. GABAergic interneurons
7. Peroxisome proliferator-activated receptor- $\gamma$
8. Carboxymethylcellulose
9. Ketamine
10. Xylazine


جدول ا. اثر ييوگليتازون بر مدتزمان (بر حسب ثانيه) لازم براى شروع مراحل مختلف تشنج ناشى از ينتيلن ترازول بر اساس معيار راسين در موش سورى

\begin{tabular}{|c|c|c|c|c|c|}
\hline مرجله ه & مرحله ع & مرحله & مرحله r & مرحله 1 & كروه \\
\hline- & - & - & - & - & كنترل \\
\hline- & - & - & - & - & يبيوكليتازون (+1ميلى كرم بر كيلوكرم، خوراكى) \\
\hline$r \mid \& / F \pm \& / M$ & $r \cdot r / r \pm s / \pi r$ & $\Delta \pm 1+\varphi / A r$ & $q+/ 4 \pm r / q 9$ & $Y \pm \Delta N+Y$ & ينتيلن تترازول (ه1 ميلى كرم بر كيلوكرم، داخل صفاقى) \\
\hline - & $\mathrm{req} / \mathrm{Y} \mathrm{V} \pm N \mathrm{NM}^{* * *}$ & $|\gamma \pm| \& \Delta /\left.\Delta\right|^{\prime}$ & $111 / f \pm N T r e=$ & 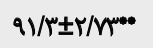 & ل \\
\hline
\end{tabular}

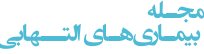

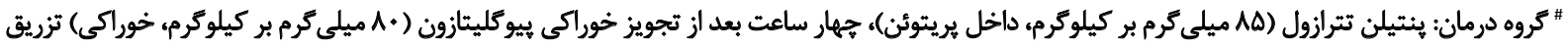

عدم وجود اختلاف بين ميانغين نتئيج بهدست آمده أز ميزان مالون

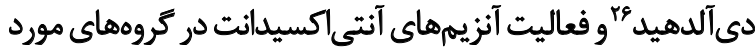

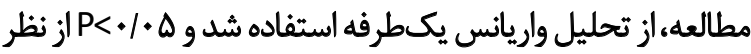
آمارى معنى دار در نظر كرفته شدل

1. dect

نتايج مطالعه نشان داد كه تجويز خوراكى بييوكليتازون

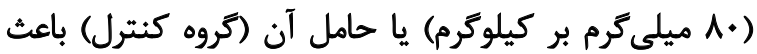

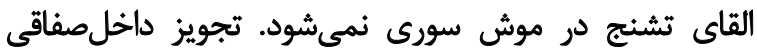

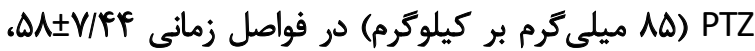

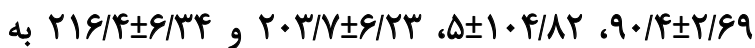
ترتيب باعث شروع مراحل اول تا ينجمم تشهنج مي شئود. تجويز

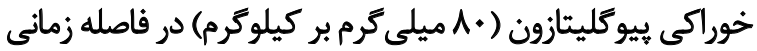

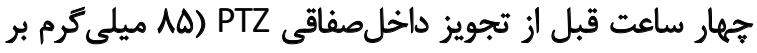

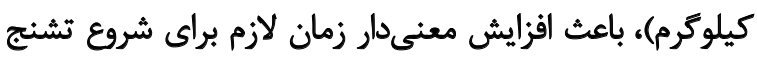

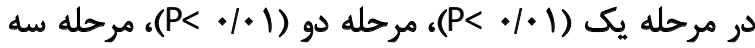

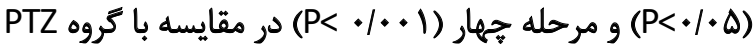

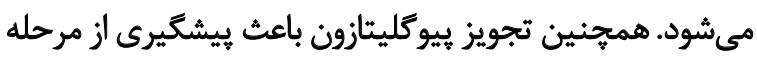

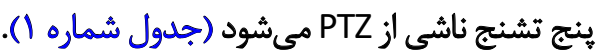

تشنج ناشى از PTZ به ميزان قابل ملاحظهاى باعث افزايش ميزان

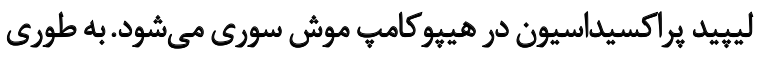

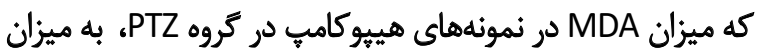

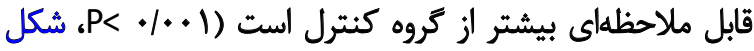

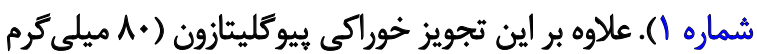

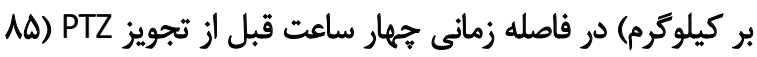

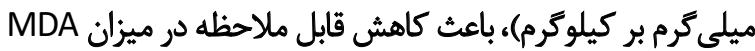

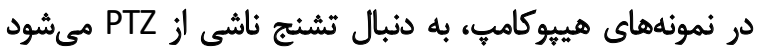

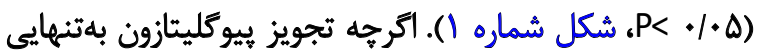

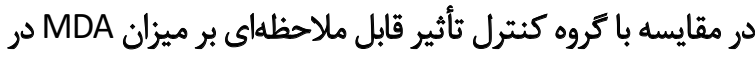

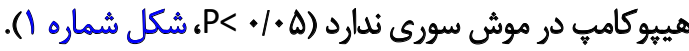

26. Malondialdehyde
(

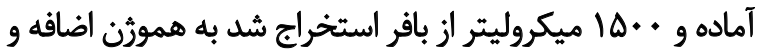

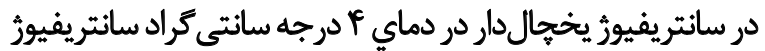

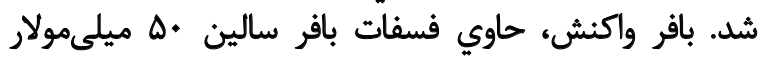

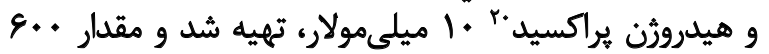

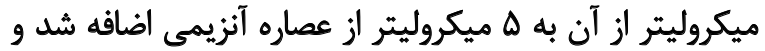

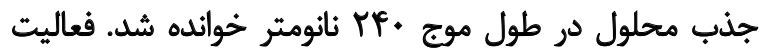

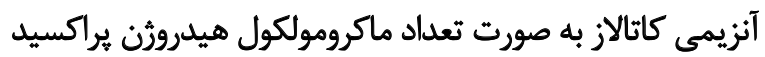

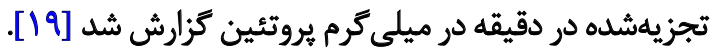

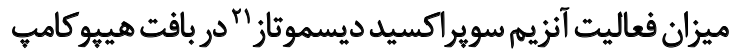

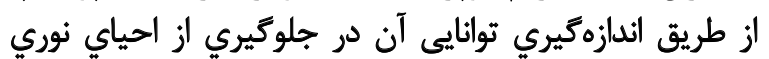

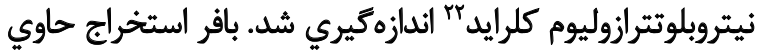

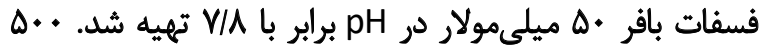

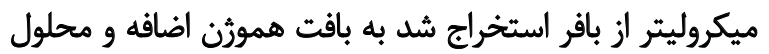

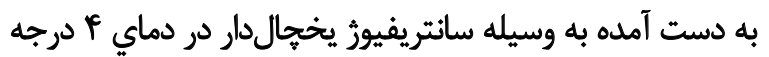

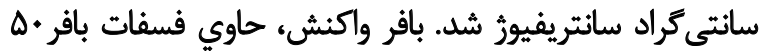

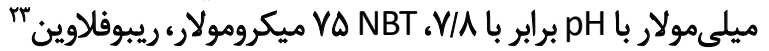

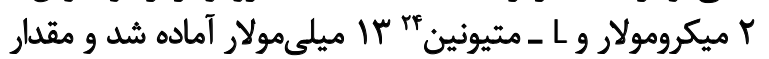

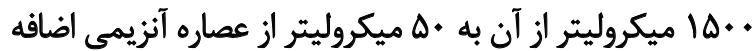

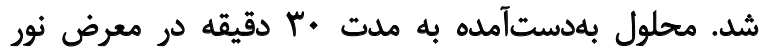
يكنواخت قرار كرفت. جذب محلول در طول موج ، بهات نانومتر

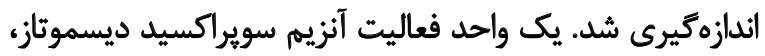

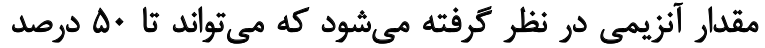

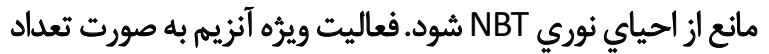

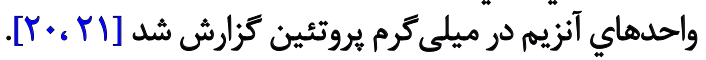
نتايج بر اساس ميانكين土انحراف معيار بيان شدند. براى

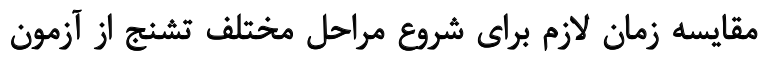

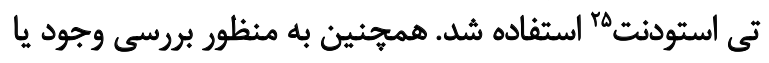

\section{Hydrogen peroxide}

21. Superoxide dismutase

22. Nitroblue tetrazolium chloride (NBT)

23. Riboflavin

24. L-methionine

25. Student t-test 


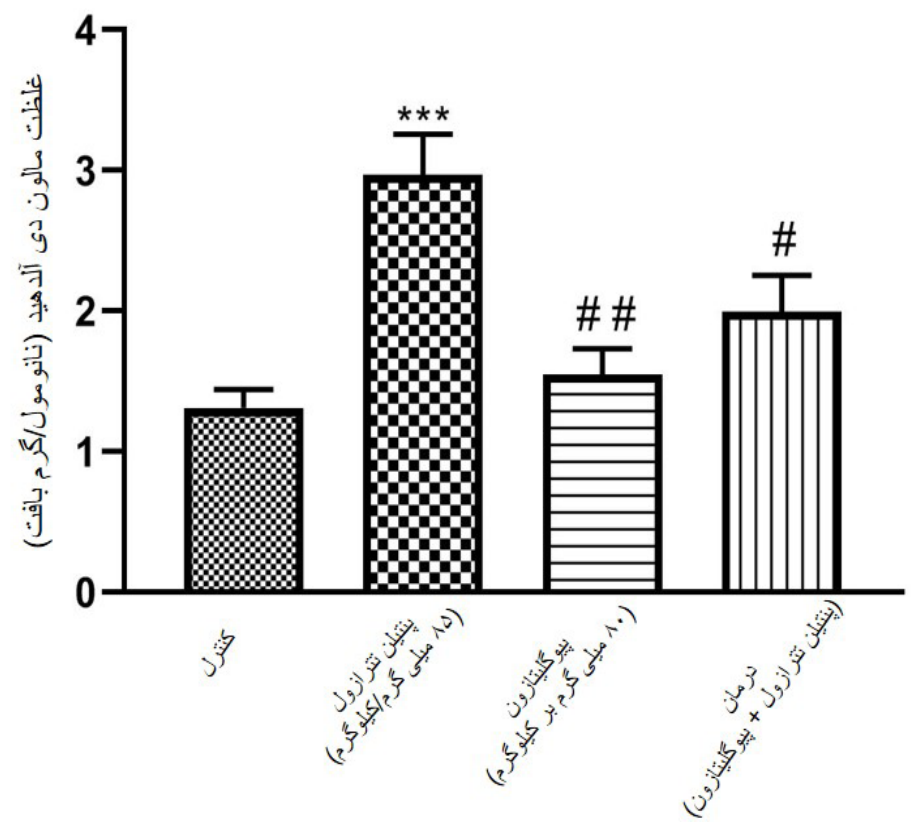

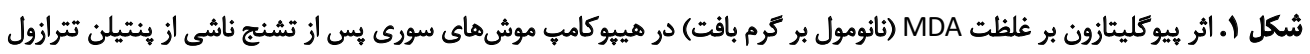

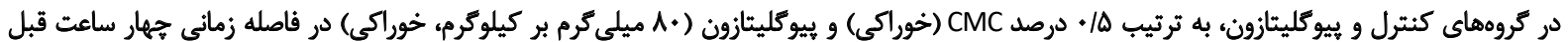

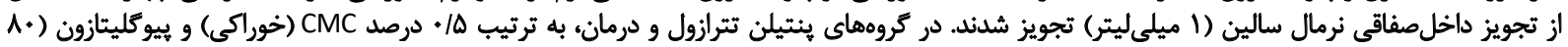

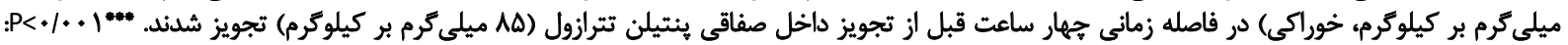

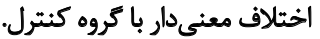

آسيب به نورون ها در قسمتهاى مختلف سيستم عصبى مركزى

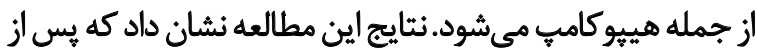

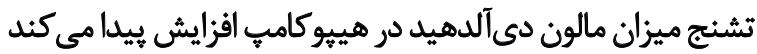

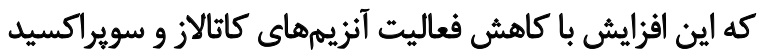
ديسموتاز همراه است.

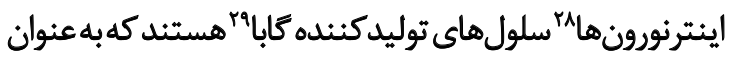

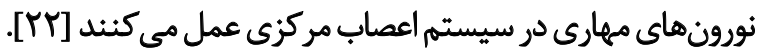

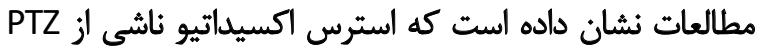

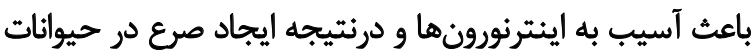

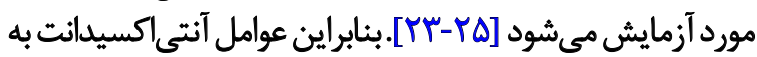

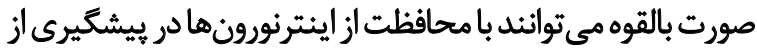

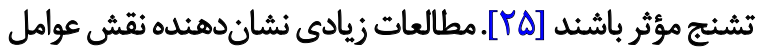

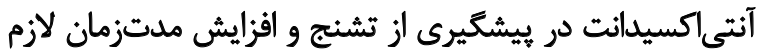

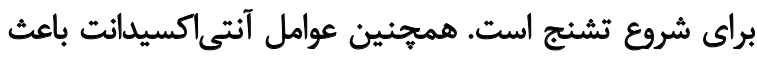
محافظت از نورونها در برابر استرس اكسيداتيو ناشى از تشنتج

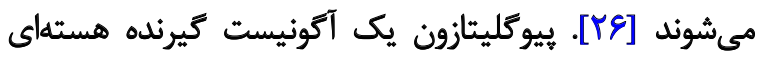
است كه باعث افزايش حساسيت كيرندهائ انسولين

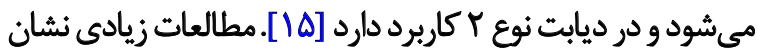

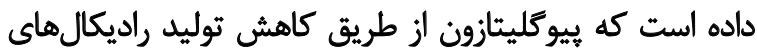

28. Interneurons

29. Gamma aminobutyric acid

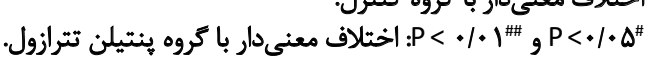

تشنج ناشى از PTZ به ميزان قابل ملاحظهاى باعث كاهش

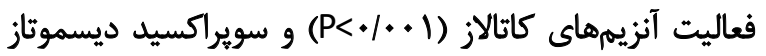

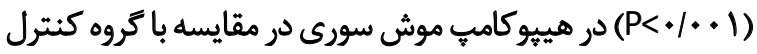

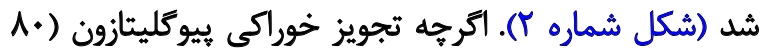

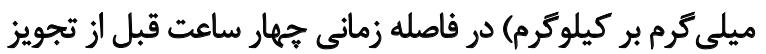

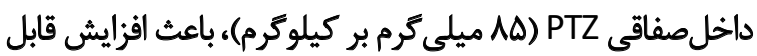

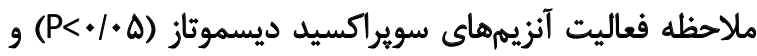

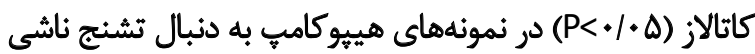

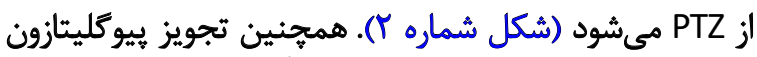

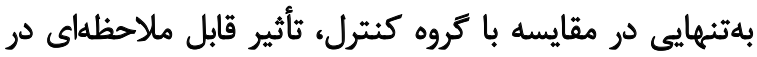

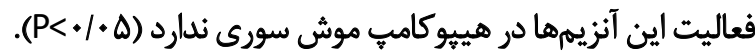

\section{s.sositg}

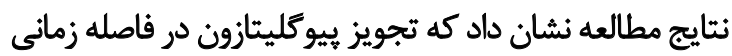

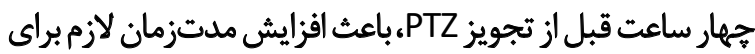

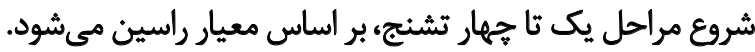

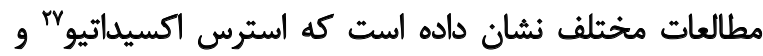

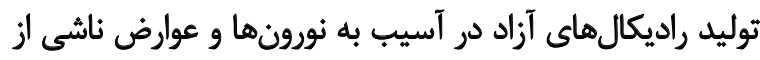

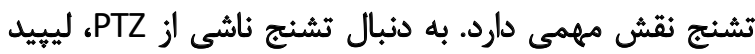

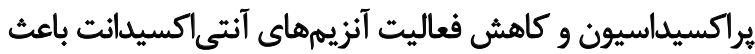



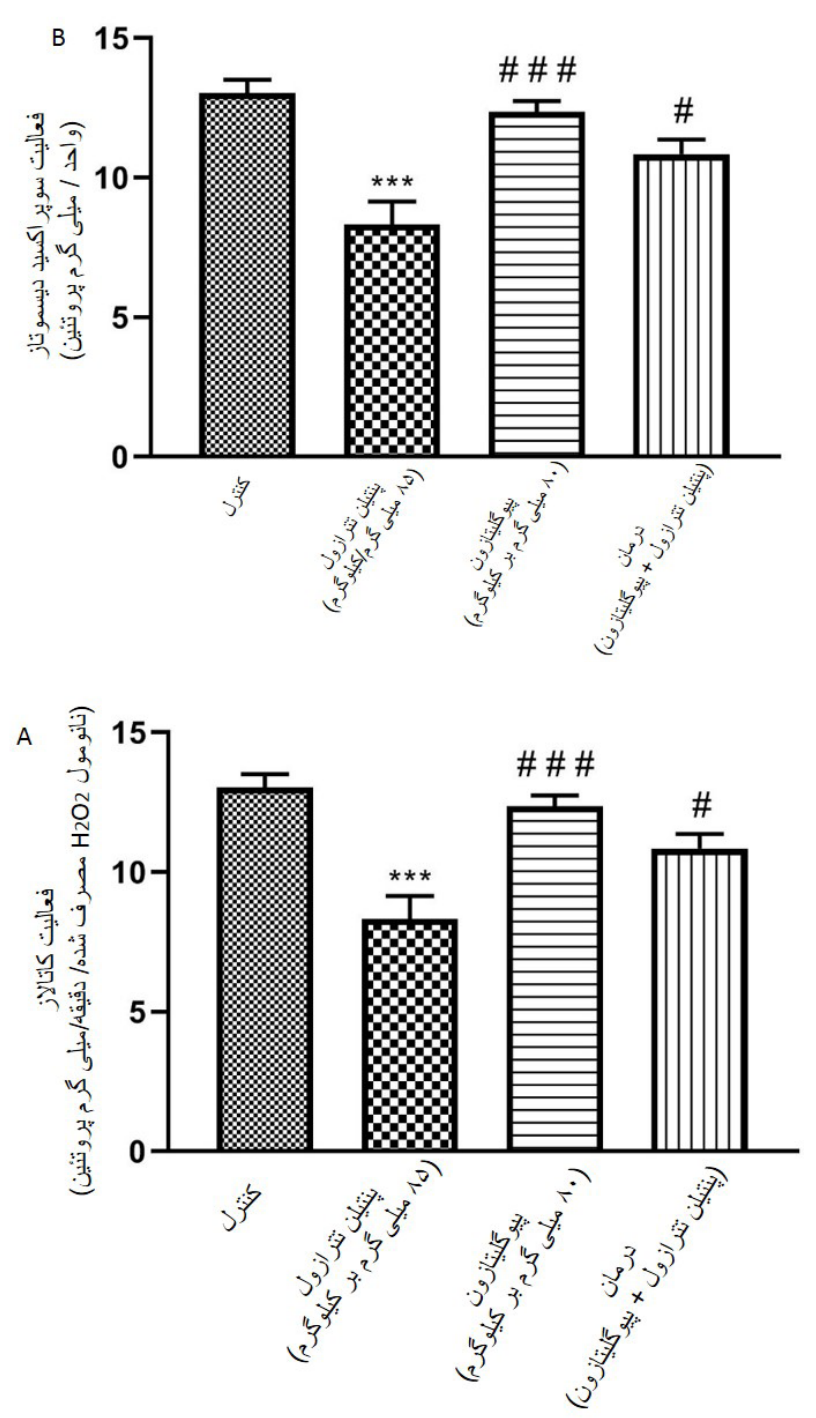

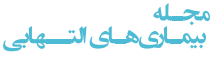

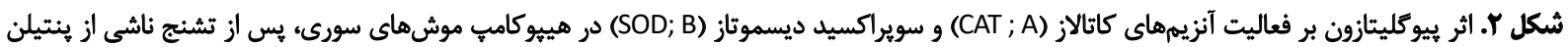

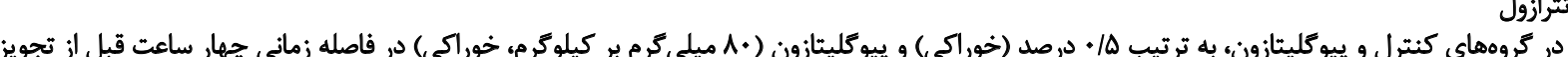

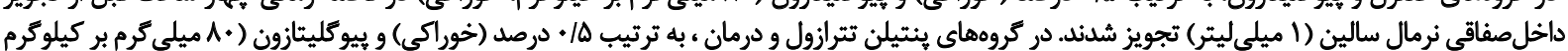

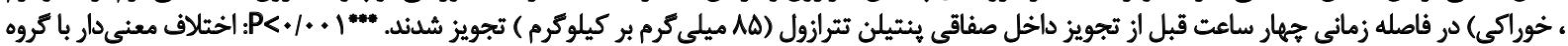

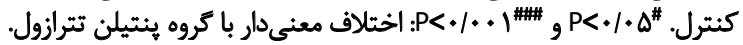

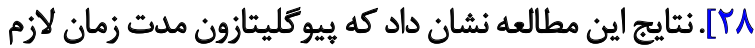

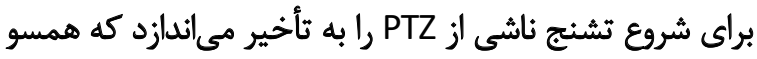

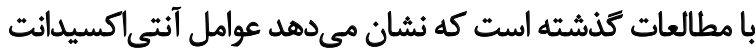

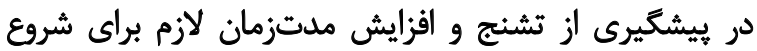

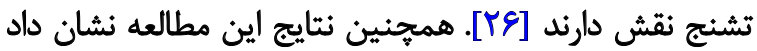

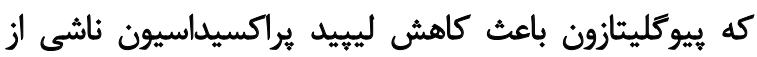

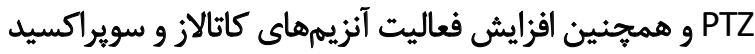

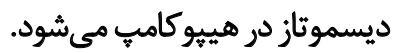

نتايج اين مطالعه نشان داد كه بيوكليتازون باعث افزايش

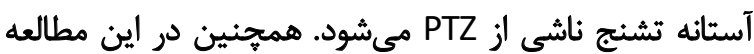

آزاد و افزايش فعاليت آنزيمهاى آنتى اكسيدانت، باعث محافظت

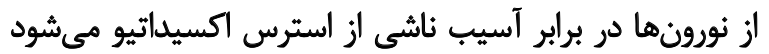
[YV]

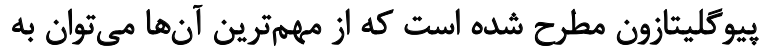

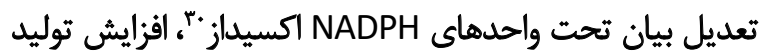
سويراكسيد ديسموتاز و مهار مسير سيكنال هاي فاكتور هستئهاي تهاي

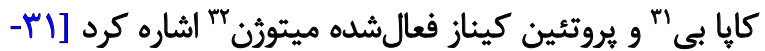

30. Nicotinamide adenine dinucleotide phosphate oxidase 31. Nuclear factor-KB (NF-KB)

32. Mitogen activated protein kinase (MAPKs) 


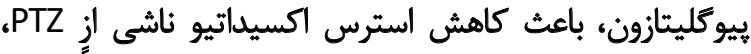

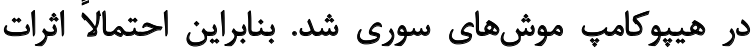

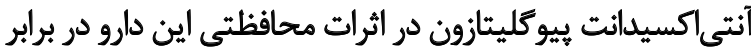

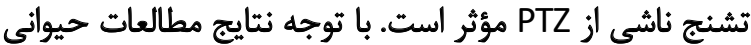

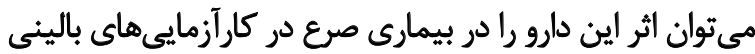

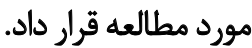

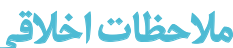

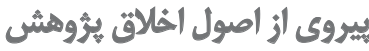
اين مطالعه با كد IR.QUMS.REC.1398.004 در كميته اخلاق دانشكاه علوميزشكى قزوين تصويب شد.

$$
\text { حامي مالى }
$$

بخشى از منابع اين بروهش از كميته تحقيقات دانشجويى

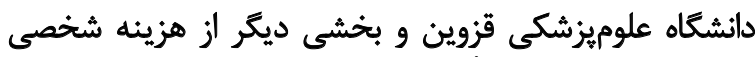

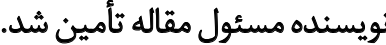

$$
\text { مشاركت نويسندكان }
$$

تمام نويسندكان در طراحي، اجرا و نكارش همه بخشهاى براي ئروهش حاضر مشاركت داشتهاند.

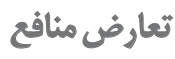

بنابر اظهار نويسندكان اين مقاله تعارض منافع ندارد. 


\section{References}

[1] Stafstrom CE, Carmant L. Seizures and Epilepsy: An overview for neuroscientists. Cold Spring Harb Perspect Med. 2015; 5(6):a022426. [DOI:10.1101/cshperspect.a022426] [PMID] [PMCID]

[2] Manford M. Recent advances in epilepsy. J Neurol. 2017; 264(8):181124. [DOI:10.1007/s00415-017-8394-2] [PMID] [PMCID]

[3] Ashjazadeh N, Yadollahikhales G, Ayoobzadehshirazi A, Sadraii $\mathrm{N}$, Hadi N. Comparison of the health-related quality of life between epileptic patients with partial and generalized seizure. Iran J Neurol. 2014; 13(2):94-100. [PMID] [PMCID]

[4] Perucca P, Mula M. Antiepileptic drug effects on mood and behavior: Molecular targets. Epilepsy Behav. 2013; 26(3):440-9. [DOI:10.1016/j.yebeh.2012.09.018] [PMID]

[5] Zhu HL, Wan JB, Wang YT, Li BC, Xiang C, He J, et al. Medicinal compounds with antiepileptic/anticonvulsant activities. Epilepsia. 2014; 55(1):3-16. [DOI:10.1111/epi.12463] [PMID]

[6] Kowski AB, Weissinger F, Gaus V, Fidzinski P, Losch F, Holtkamp $M$. Specific adverse effects of antiepileptic drugs -- A trueto-life monotherapy study. Epilepsy Behav. 2016; 54:150-7. [DOI:10.1016/j.yebeh.2015.11.009] [PMID]

[7] Wahab A. Difficulties in treatment and management of epilepsy and challenges in new drug development. Pharmaceuticals (Basel). 2010; 3(7):2090-110. [DOI:10.3390/ph3072090] [PMID] [PMCID]

[8] Ono T, Galanopoulou AS. Epilepsy and epileptic syndrome. Adv Exp Med Biol. 2012; 724:99-113. [DOI:10.1007/978-1-46140653-2_8] [PMID] [PMCID]

[9] Gu B, Daltone KA. Models and detection of spontaneous recurrent seizures in laboratory rodents. Zool Res. 2017; 38(4):1719. [DOI:10.24272/j.issn.2095-8137.2017.042] [PMID] [PMCID]

[10] Geronzi U, Lotti F, Grosso S. Oxidative stress in epilepsy. Expert Rev Neurother. 2018; 18(5):427-34. [DOI:10.1080/14737175.20 18.1465410] [PMID]

[11] Pearson-Smith JN, Patel M. Metabolic dysfunction and oxidative stress in epilepsy. Int J Mol Sci. 2017; 18(11):2365. [DOI:10.3390/ijms18112365] [PMID] [PMCID]

[12] Martinc B, Grabnar I, Vovk T. Antioxidants as a preventive treatment for epileptic process: A review of the current status. Curr Neuropharmacol. 2014; 12(6):527-50. [DOI:10.2174/1570 159X12666140923205715] [PMID] [PMCID]

[13] Shimada T, Yamagata K. Pentylenetetrazol-induced kindling mouse model.JVis Exp. 2018; (136):56573. [DOI:10.3791/56573] [PMID] [PMCID]

[14] Zaitsev AV. The role of GABAergic interneurons in cortex and hippocampus in the development of epilepsy. Neurosci Behav Physiol. 2017; 47:913-22. [DOI:10.1007/s11055-017-0491-2]

[15] Pavlova V, Filipova E, Uzunova K, Kalinov K, Vekov T. Pioglitazone therapy and fractures: Systematic review and metaanalysis. Endocr Metab Immune Disord Drug Targets. 2018; 18(5):502-7. [DOI:10.2174/1871530318666180423121833] [PMID] [PMCID]
[16] Karabas MK, Ayhan M, Guney E, Serter M, Meteoglu I. The effect of pioglitazone on antioxidant levels and renal histopathology in streptozotocin-induced diabetic rats. ISRN Endocrinol. 2013; 2013:858690. [DOI:10.1155/2013/858690] [PMID] [PMCID]

[17] Cela E, McFarlan AR, Chung AJ, Wang T, Chierzi S, Murai KK, et al. An optogenetic kindling model of neocortical epilepsy. Sci Rep. 2019; 9(1):5236. [DOI:10.1038/s41598-019-41533-2] [PMID] [PMCID]

[18] Ghani MA, Barril C, Bedgood DR Jr, Prenzler PD. Measurement of antioxidant activity with the thiobarbituric acid reactive substances assay. Food Chem. 2017; 230:195-207. [DOI:10.1016/j. foodchem.2017.02.127] [PMID]

[19] Hadwan MH. Simple spectrophotometric assay for measuring catalase activity in biological tissues. BMC Biochem. 2018; 19(1):7. [DOI:10.1186/s12858-018-0097-5] [PMID] [PMCID]

[20] Durak I, Yurtarslanl Z, Canbolat O, Akyol O. A methodological approach to Superoxide Dismutase (SOD) activity assay based on inhibition of Nitroblue Tetrazolium (NBT) reduc tion. Clin Chim Acta. 1993; 214(1):103-4. [DOI:10.1016/0009 8981(93)90307-P] [PMID]

[21] Carlberg I, Mannervik B. Glutathione reductase. In: Meister A. Glutamate, Glutamine, Glutathione, and Related Compounds. Methods in Enzymology. Vol. 113. Academic Press: Cambridge, MA: 1985. p. 484-490. [DOI:10.1016/S0076-6879(85)13062-4] [PMID]

[22] Katsarou AM, Moshé SL, Galanopoulou AS. Interneuronopathies and their role in early life epilepsies and neurodevelopmental disorders. Epilepsia Open. 2017; 2(3):284-306. [DOI:10.1002/epi4.12062] [PMID] [PMCID]

[23] Gao R, Penzes P. Common mechanisms of excitatory and inhibitory imbalance in schizophrenia and autism spectrum disorders. Curr Mol Med. 2015; 15(2):146-67. [DOI:10.2174/1566 524015666150303003028] [PMID] [PMCID]

[24] Dudek FE, Shao LR. Loss of GABAergic interneurons in seizureinduced epileptogenesis. Epilepsy Curr. 2003; 3(5):159-61. [DOI:10.1046/j.1535-7597.2003.03503.x] [PMID] [PMCID]

[25] Faghihi N, Mohammadi MT. Anticonvulsant and antioxidant effects of pitavastatin against pentylenetetrazol-induced kindling in mice. Adv Pharm Bull. 2017; 7(2):291-8. [DOI:10.15171/ apb.2017.035] [PMID] [PMCID]

[26] Puttachary S, Sharma Sh, Stark S, Thippeswamy T. Seizureinduced oxidative stress in temporal lobe epilepsy. Biomed Res Int. 2015; 2015:745613. [DOI:10.1155/2015/745613] [PMID] [PMCID]

[27] Lee CH, Yi MH, Chae DJ, Zhang E, Oh SH, Kim DW. Effect of pioglitazone on excitotoxic neuronal damage in the mouse hippocampus. Biomol Ther (Seoul). 2015; 23(3):261-7. [DOI:10.4062/biomolther.2014.146] [PMID] [PMCID]

[28] Chandrasekar T, Muthiah NS, Sandiya R, Sanitha, Aparna. Evaluation of antioxidant activity of pioglitazone: Hydrogen peroxide scavenging activity (in-vitro method). Res J Pharm Biol Chem Sci. 2015; 6(5):1492-5. https://www.rjpbcs.com/ pdf/2015_6(5)/[206].pdf 
(Previous Title: The Journal of Qazvin University of Medical Sciences)

[29] Pérez-Girón JV, Palacios R, Martín A, Hernanz R, Aguado A, Martínez-Revelles $\mathrm{S}$, et al. Pioglitazone reduces angiotensin IIinduced COX-2 expression through inhibition of ROS production and ET-1 transcription in vascular cells from spontaneously hypertensive rats. Am J Physiol Heart Circ Physiol. 2014; 306(11):H1582-93. [DOI:10.1152/ajpheart.00924.2013] [PMID]

[30] Wang Sh, Ye SD, Sun WJ, Hu YY. Pioglitazone inhibits the expressions of p22phox and p47phox in rat mesangial cells in vitro. ISRN Endocrinol. 2014; 2014:601352 [DOI:10.1155/2014/601352] [PMID] [PMCID]

[31] Jesse CR, Bortolatto CF, Wilhelm EA, Roman SS, Prigol $\mathrm{M}$, Nogueira CW. The peroxisome proliferator-activated receptor- $\gamma$ agonist pioglitazone protects against cisplatin-induced renal damage in mice. J Appl Toxicol. 2014; 34(1):25-32. [DOI:10.1002/jat.2818] [PMID] 
This Page Intentionally Left Blank 